\title{
Nocturnal convulsions and insulin-induced hypoglycaemia in diabetic patients
}

\author{
B. Miles Fisher and Brian M. Frier, \\ Diabetic Department, Gartnavel General Hospital and Western Infirmary, Glasgow G12 OYN, UK.
}

\begin{abstract}
Summary: Convulsions may occur as a consequence of insulin-induced hypoglycaemia. We report three patients with insulin-dependent diabetes, who presented with generalized tonic-clonic seizures associated with nocturnal hypoglycaemia. None of the patients had experienced hypoglycaemia during waking hours and the convulsions were mistakenly diagnosed as idiopathic epilepsy. Recognition of the possible hypoglycaemic aetiology of these convulsions permitted appropriate alteration of the insulin regimens with no recurrence of convulsions. In one case, the seizure was associated with bilateral fractures of the neck of the humerus. Unrecognized hypoglycaemia should be considered as a possible cause of convulsions in insulin-dependent diabetic patients.
\end{abstract}

\section{Introduction}

Hypoglycaemia is a common problem in diabetic patients treated with insulin. The neurological consequences of hypoglycaemia include impairment of mentation and cognitive functions, ${ }^{1}$ depression, aggressive or irrational behaviour, confusion, transient ischaemic attacks, hemiplegia, convulsions and coma. ${ }^{2}$ When hypoglycaemia occurs during sleep the patient is often unaware of the autonomic warning symptoms, and convulsions induced by hypoglycaemia may be mistakenly diagnosed as idiopathic epilepsy. We describe three patients with insulindependent diabetes, who presented with generalized, tonic-clonic seizures associated with nocturnal hypoglycaemia. None of the patients had experienced hypoglycaemia during waking hours, nor had a previous history of fits or blackouts.

\section{Case reports}

Case 1

A 31 year old unmarried engineer with insulin-dependent diabetes for 12 years complained of severe tenderness, bruising, and restricted mobility of his shoulders after awakening from sleep. X-rays revealed a fracture of the neck of each humerus which required orthopaedic surgery (Figure 1). In the absence of a preceding history of trauma, an epileptic seizure was suspected. Investigations included a normal isotope brain scan and an electroencephalogram (EEG) per-

Correspondence: B.M. Fisher, M.B., M.R.C.P.

Accepted: 19 February 1987 formed 2 days after presentation, which showed pronounced theta slow wave activity over the left hemisphere. He was receiving treatment with twice daily soluble and isophane insulins, in a total dose of 88 units per day (1.2 units $/ \mathrm{kg}$ body weight). A recent glycosylated haemoglobin was $6.4 \%$ (normal, nondiabetic range: $5-8 \%$ ). His glycaemic control appeared to be satisfactory in hospital, his insulin dose was not changed, and anticonvulsant therapy was not started.

Two months later he suffered a witnessed nocturnal convulsion, during which he bit his tongue and had transient post-ictal aphasia. A capillary blood glucose [BM-Test-Glycemie (1-44) Strips, BCL] which was measured the following morning at 09.00 hours, was $4 \mathrm{mmol} / \mathrm{l}$. Anti-convulsant therapy in the form of carbamazepine was commenced by a neurologist, and the patient's driving licence was revoked by the Driver and Vehicle Licensing Centre (DVLC). He experienced two further episodes of nocturnal hypoglycaemia without convulsions, both of which were treated with intramuscular glucagon, administered by a relative. Capillary blood glucose was $1 \mathrm{mmol} / 1$ during each hypoglycaemic episode. He reported nocturnal sweating and a morning 'hang-over' and subsequent selfmonitoring of capillary blood glucose through the night showed profound falls to $1 \mathrm{mmnl}^{\prime \prime}$ hetween 04.00 and $06.00 \mathrm{~h}$. His evening dose of isophane insulin was reduced, following which he had neither nocturnal hypoglycaemia nor convulsions. Anticonvulsant therapy was stopped and a subsequent EEG was normal. His driving licence was reinstated after a period of restabilization, during which he experienced no further hypoglycaemia.

C The Fellowship of Postgraduate Medicine, 1987 


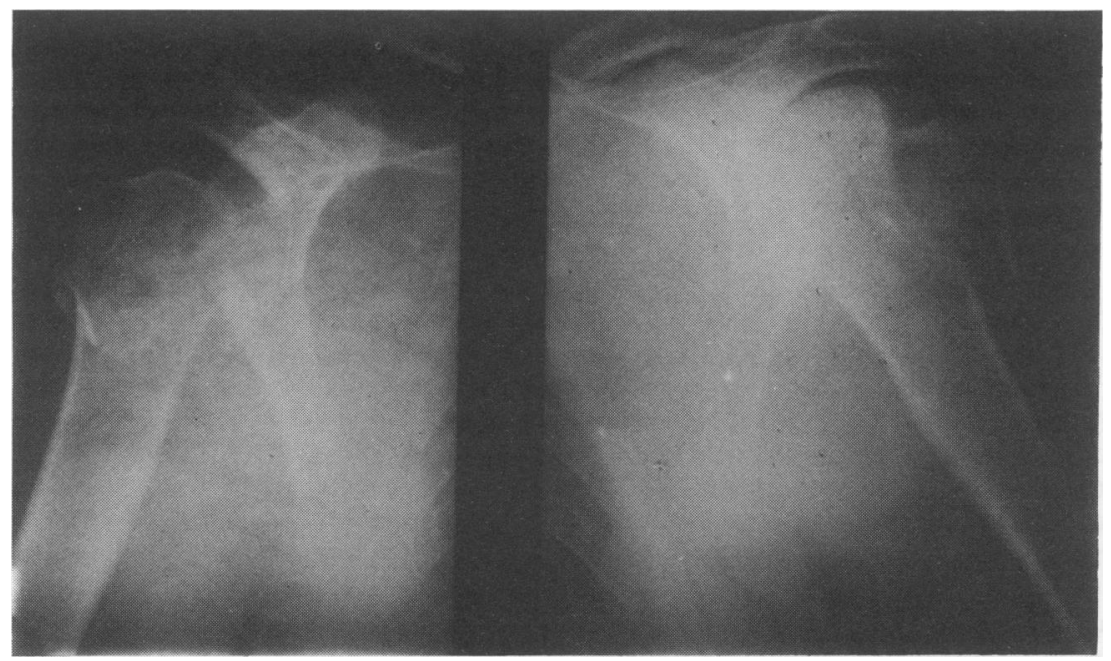

Figure 1 Case 1: fractures of neck of humerus in right and left arms following presumed convulsion.

Case 2

A 29 year old housewife with insulin-dependent diabetes for 4 years suffered two separate nocturnal tonic-clonic seizures. An isotope brain scan was normal, but an EEG showed a focal disturbance in the right temporo-occipital area. On each occasion capillary blood glucose (BM Strips) was estimated 2 hours after recovery and was approximately $4 \mathrm{mmol} / \mathrm{l}$. She was receiving treatment with short and intermediate-acting insulins twice daily, in a total dose of 40 units $(0.7 \mathrm{U} / \mathrm{kg})$, on which regimen her glycaemic control was excellent with a glycosylated haemoglobin of $7.3 \%$. The patient was asked to perform serial measurements of capillary blood glucose during the night at home, which revealed values of less than $2 \mathrm{mmol} / 1$ around $04.00 \mathrm{~h}$, with fasting hyperglycaemia of $13 \mathrm{mmol} / 1$ at $08.00 \mathrm{~h}$. Anticonvulsant therapy was not prescribed, but her evening dose of insulin was reduced. Subsequently, she had neither hypoglycaemic attacks nor convulsions.

\section{Case 3}

A 24 year old salesman with insulin-dependent diabetes for 10 years was admitted to hospital following a nocturnal tonic-clonic convulsion. His wife, suspecting that he was hypoglycaemic, injected glucagon intramuscularly during the convulsion which was terminated successfully. Two years previously he had suffered a similar tonic-clonic seizure during the night. An EEG had demonstrated bilateral slow wave abnormalities, and anticonvulsant therapy had been commenced without any alteration to his insulin dose or regimen. His total daily dose 306 twice daily soluble and isophane insulins was 52 unts $(0.66 \mathrm{U} / \mathrm{kg})$, giving optimal glycaemic control, wit glycosylated haemoglobin of $6.9 \%$. In the two years after reducing the evening dose of isophane insulin he has experienced one isolated episode of severe daytime hypoglycaemia requiring intravenous dextrose. $\mathrm{H}_{\varrho}^{\mathrm{D}}$ has had no further epileptiform seizures and anticon $=\frac{\vec{F}}{\overrightarrow{7}}$ vulsant therapy has been stopped.

\section{Discussion}

Convulsions are a well recognized complication of acute hypoglycaemia in insulin-dependent diabetic. patients, but idiopathic epilepsy is considered to be nog more common in the diabetic than in the non-diabetic population. ${ }^{3,4}$ It has been suggested that the pathogen 0 esis of all convulsions in diabetic patients treated with insulin should be regarded as being caused byo hypoglycaemia until proved otherwise. This would avoid unnecessary treatment with anticonvulsantos therapy as demonstrated by two of our case histories N The mechanism by which a convulsion is precipitated is uncertain, but it may be provoked by a sudden rise in brain osmolality and a net influx of electrolytes, which have been attributed to a direct effect of insulin. ${ }^{5}$ The prophylactic value of anticonvulsant therapy to prevent hypoglycaemic-induced seizures is undeter:mined. The frequency of bone fractures following 
hypoglycaemia-induced convulsions is not known. Compression fractures of vertebral bodies have been described following nocturnal hypoglycaemic seizures. ${ }^{6}$ One patient in that reported series had no recollection of the hypoglycaemic episode, but presented with back pain in a similar manner to Case 1.

Electroencephalography is frequently performed in any patient with suspected convulsions. However, the presence of EEG abnormalities may be misleading in diabetic patients, especially if recorded within a few days of the convulsion. Acute hypoglycaemia is associated with pronounced changes of the EEG without the development of convulsions, ${ }^{7}$ and generalized slowing of the EEG becomes evident when the blood glucose falls below $2.6 \mathrm{mmol} / 1$ in diabetic and non-diabetic subjects. ${ }^{8}$ It is important therefore to measure blood glucose at the time of an EEG examination in a patient with insulin-dependent diabetes to exclude concurrent hypoglycaemia. Diabetic patients subjected to repeated hypoglycaemia may have an abnormal EEG between the hypoglycaemic episodes. ${ }^{4}$ In Case 1 the decision to commence anticonvulsant therapy was influenced by the abnormal EEG recorded in the immediate post-ictal phase. It is not known for how long EEG abnormalities persist following a hypoglycaemic convulsion; the EEG eventually returned to normal in our three patients after suitable modification of their insulin regimes to avoid hypoglycaemia.

Nocturnal hypoglycaemia is common, but is often unrecognized in insulin-dependent diabetic patients. ${ }^{9,10}$ Hypoglycaemic symptoms during the night are frequently mild or absent, although patients occasionally describe night sweats, nightmares, difficulty in awakening and early morning headache or 'hangover'. Patients complain of chronic fatigue and a poor quality of sleep. These symptoms in a diabetic patient should alert the physician to the possibility of nocturnal hypoglycaemia. Although the sleeping patient is usually oblivious to the autonomic warning symptoms of hypoglycaemia, a spouse or relative may be aroused by restless behaviour or sweating. Nocturnal hypoglycaemia was unrecognized in Cases 1 and 2 before the episodes which provoked convulsions and in all three patients the convulsions occurred only during sleep. Because none of the patients had a previous history of serious hypoglycaemia, they were assumed to have developed idiopathic epilepsy. In a previous study of insulin-dependent diabetic patients, nocturnal hypoglycaemia associated with a history of convulsions was identified in five patients, in whom a diagnosis of idiopathic epilepsy had been made. ${ }^{9}$ Further investigation confirmed a diagnosis of idiopathic epilepsy in two of these five patients, but the convulsions ceased following a reduction of insulin dosage in the other three patients in whom anticonvulsant therapy was stopped successfully, and the EEG reverted to normal.

Accurate diagnosis of epilepsy or hypoglycaemiainduced seizures is of considerable medico-legal importance, particularly with respect to driving. A driving licence which has been revoked because of epileptic fits, will not be restored until 2 years have elapsed during which the patient has no convulsions. The DVLC are prepared to restore the driving licence to an insulin-dependent diabetic driver after a shorter time interval if isolated convulsions can be attributed to hypoglycaemia, and therapeutic alterations have successfully prevented recurrence. Similarly, nocturnal convulsions caused by hypoglycaemia which occur during sleep have a less serious import on medical fitness to drive.

The apparent increase in the frequency of severe nocturnal hypoglycaemia in insulin-treated patients may result from increasing use of intensive insulin regimens and the current emphasis on attaining good glycaemic control. Hypoglycaemia is more frequent in diabetic patients who have optimal glycaemic control and a low glycosylated haemoglobin within the normal range." In all three of the patients described, glycaemic control was super-optimal as estimated by glycosylated haemoglobin concentrations. The identification of nocturnal hypoglycaemia is often difficult, ${ }^{12}$ but it is now relatively simple for patients to measure capillary blood glucose at suitable times during the night. ${ }^{13}$ The avoidance of subclinical hypoglycaemia during the night may require the occasional random measurement of capillary blood glucose by diabetic patients as part of their routine of blood glucose monitoring at home. This may 'awaken' both the patient and the doctor to the reality of nocturnal hypoglycaemia.

\section{Acknowledgement}

We thank Miss Alison Wood for expert secretarial assistance.

(eds) Hypoglycaemia, 2nd Edition. Blackwell Scientific Publications, Oxford, 1981, pp90-109.

3. Eeg-Olofsson, O. Hypoglycaemia and neurological disturbance in children with diabetes mellitus. Acta Paediatr Scand 1977, 270 (Suppl): 91-96. 
4. Haumont, D., Dorchy, H. \& Pell, S. EEG abnormalities in diabetic children. Clin Pediatr (Phil) 1979, 18: 750753.

5. Arieff, A.I., Doerner, T., Zelig, H. \& Massry, S.G. Mechanisms of seizures and coma in hypoglycemia. Evidence for a direct effect of insulin on electrolyte transport in brain. J Clin Invest 1974, 54: 654-663.

6. Nabarro, J.D.N. Compression fractures of the dorsal spine in hypoglycaemic fits in diabetes. $\mathrm{Br}$ Med $\mathrm{J} 1985$, 291: 1320.

7. Hoagland, H., Rubin, M.A. \& Cameron, D.E. The EEG of schizophrenics during insulin hypoglycemia recovery. Am J Physiol 1937, 120: 559- 570

8. Harrad, R.A., Cockram, C.S., Plumb, A.P., Stone, S., Fenwick, P. \& Sonksen, P.H. The effect of hypoglycaemia on visual function: a clinical and electrophysiological study. Clin Sci 1985, 69: 673-679.
9. Gale, E.A.M. \& Tattersall, R.B. Unrecognised nocturna hypoglycaemia in insulin-treated diabetics. Lancet 1979 i: $1049-1052$.

10. Pramming, S., Thorsteinsson, B., Bendtson, I., Ronn, B. \& Binder, C. Nocturnal hypoglycaemia in patients receiving conventional treatment with insulin. $\mathrm{Br}$ Med $f$ ? 1985. 291: 376-379.

11. Unger, R.H. Meticulous control of diabetes: benefits risks and precautions. Diabetes 1982, 31: 479-483.

12. Silas, J.H., Grant, D.S. \& Maddocks, J.L. TransientD hemiparetic attacks due to unrecognised nocturnaf hypoglycaemia. $\mathrm{Br}$ Med J 1981, 282: 132-133.

13. Sonksen, P.H., Judd, S. \& Lowy, C. Home monitoring of blood glucose: new approach to management of insulin:dependent diabetic patients in Great Britain. Diabetes Care 1980, 3: 100-107. 